\title{
Effortless attention and composite challenges in movement interaction
}

\author{
Pablo Romero \\ IIMAS, Universidad Nacional \\ Autónoma de México (UNAM) \\ México \\ pablor@unam.mx
}

\author{
Eduardo Calvillo-Gámez \\ Universidad Polítecnica \\ de San Luis Potosí, \\ México \\ eduardo.calvillo@gmail.com
}

\begin{abstract}
This paper describes an empirical investigation into the nature of challenge within the context of flow or effortless attention. An important condition of the flow state is that the challenges of the task are commensurate with the person's level of skill. However when considering movement interaction those challenges could be composite in the sense that they could comprise physical as well as intellectual elements. In order to evaluate the importance of composite challenges, this investigation compares an unbalanced with a balanced activity in terms of their challenge composition. The results of the study suggest that balanced activities are more likely to promote flow but also highlight the need of undertaking more detailed studies of balancing in composite challenges within the context of flow.
\end{abstract}

Flow, User Experience, Movement Interaction

\section{INTRODUCTION}

The concept of flow has been used to describe psychological states of optimal experience that are characterised by a deep concentration in the task at hand, a merging of action and awareness, a loss of self-consciousness, a sense of control, time distortion and experiencing the activity as intrinsically rewarding (Csikszentmihalyi and Nakamura 2010). Flow has been associated with intrinsic motivation, skills promotion and academic excellence (Nakamura and Csikszentmihalyi 2002). Computing studies of this concept have reported digital environments as conducive to flow and promoting positive attitudes and outcomes for users (Finneran and Zhang 2005; Hoffman and Novak 2009; Voiskounsky 2008). In those studies the task at hand has been considered as cognitive mainly, perhaps because of the characteristics of conventional interaction. One of the main conditions of flow is that the task at hand promotes a balance between its challenges and the person's level of skill; if the level of challenge is too low the task might become boring, if it is too high the person could experience frustration. An appropriate level of challenge would make the person just about manage the task (Nakamura and Csikszentmihalyi 2002).
When considering movement interaction, challenges could be composite in the sense that they could comprise physical as well as intellectual elements. For example learning a dance routine in a movement interaction game has a physical element of coordination as well as a more intellectual element of kinesthetic memory. The activity might require one mode of the interaction to dominate over the other, altering the balance of the composite challenge. Although some studies have acknowledged that challenges might be composite (Finneran and Zhang 2005), as far as we are aware, balance in composite challenges and skills within the context of flow is yet to be studied. We argue that within a movement interaction context, considering challenges as balanced is of central relevance for applications trying to promote flow states. In order to evaluate the importance of balance in challenges, this study employs a movement interaction game and compares a balanced with an unbalanced activity in terms of their potential to promote states of flow.

\subsection{Flow and Balanced Composite Challenges}

In Romero and Calvillo-Gamez (2011), we propose a view of flow based on notions of phenomenology and embodied interaction (Dourish 2001). This view stresses the importance of four main points: the importance of effortless attention, the context where interaction takes place, whether the task is directly 


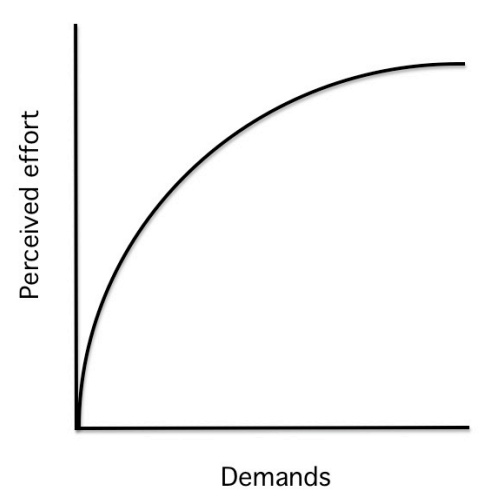

a)

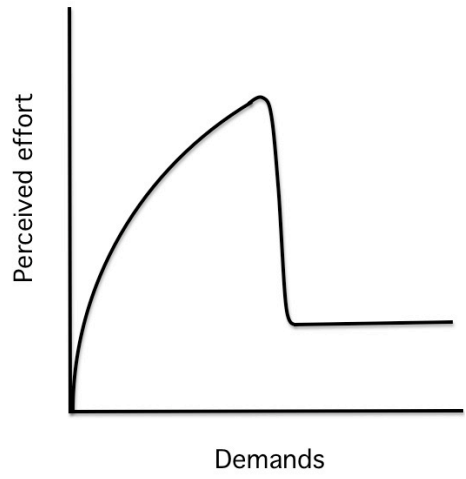

b)

Figure 1: Perceived effort vs. demands in a) effortful and b) effortless attention. From Bruya (2010).

concerned with the digital system or with any other aspect of reality, and the body and its role in the interaction with the system. The first point is very relevant for this study. Computing studies of flow have largely ignored a central characteristic of this construct: effortless attention (Csikszentmihalyi and Nakamura 2010). We develop our view around this characteristic and conceptualise flow as a state of deep concentration that is perceived as effortless. People perceive this experience as their attention being effortlessly carried by a current, hence the analogy with flow. Under ordinary circumstances, subjective attentional effort in a task is proportional to the demands of the task, until there comes a point in which no increase in effort is possible (see Figure 1a) (Bruya 2010). In contrast to this effortful attention scenario, there are occasions in which, at some point in the execution of the task, one is concentrated so thoroughly in the activity that suddenly attention seems effortless. At these moments, increased demands can be met with a sustained level of efficacy but without an increase in the perceived attentional effort (see Figure 1b).

So effortless attention, and therefore flow, is a paradox because the demands of the task would require a level of attention that should be perceived as effortful and yet the subjective experience is one of effortlessness. This does not mean that the person is paying less attention; the level of attention is high but the perceived effort to achieve it is relatively low. Here it is important to clarify what is understood by effortful attention.

According to Schmeichel and Baumeister (2010), attention can be either driven by the person doing the attending or by external stimuli (external stimuli could be internal to the person's body, as in a sensation of hunger for example). Attention can be (and constantly is) captured by external stimuli: smells, noises and images of the external world, for example. People pay attention to these stimuli without trying; in other words, their attention is grabbed without them spending any effort. In such situations we can talk about a captive attention, as there is a passive quality to it; the person is not in control of which stimuli are attended to. In contrast, sometimes people might focus and maintain attention on specific stimuli intentionally. In these cases, it is said that some effort is required to keep such focus and therefore there is a more active quality to attention. Effortful attention is said to be a specific instance of selfcontrol because the tendency to attend to constant external distractors must be overridden in order to keep an intentional focus on the chosen stimuli. Schmeichel and Baumeister (2010) differentiation between attention driven either by the person or by external stimuli is similar to the distinction between willed (or controlled) and automatic attention (Norman and Shallice 1986; Schneider and Shiffrin 1977). Besides psychology, other traditions have known and employed this distinction in their conceptual constructs and methods. For example, the experience of captive attention is strongly related to the state of not being present as understood in the Buddhist tradition of mindfulness (Varela et al. 1991).

Normally, a state of effortless attention is reached after a period of struggle or effortful attention (as illustrated in Figure 1b). It is as if only by stretching our attentional resources that the mind can arrive at a state in which we can really be masters of our own attention. It seems this struggle is required because, if unengaged, the mind tends to wander aimlessly (Csikszentmihalyi 1990; Csikszentmihalyi and Nakamura 2010; Smallwood et al. 2007; Smallwood and Schooler 2006; Varela et al. 1991), preventing any real possibility of focus and internal control. 
For a conceptualisation of flow as effortless attention considering challenges is crucial because effortless attention implies a struggle with challenges. Some other conceptualisations of flow consider challenges as an optional condition (Skadberg and Kimmel 2004; Chen 2006; Shin 2006; Novak et al. 2003) and therefore the analysis of challenges is not high on their agenda (Romero and Calvillo-Gamez 2011).

In order for a task to engage the person's attention so thoroughly, it needs to promote an appropriate level of demand; a balance between challenges and skills is key to achieve this. Studies of flow generally measure challenges and skills using unidimensional scales, yet according to Ellis et al. (1994), they might be complex multidimensional constructs in the sense that they are associated with the cognitive, physical and emotional parts of the person. In the view of flow we have proposed (Romero and Calvillo-Gamez 2011), a key topic for research is the analysis of the balance of challenges and skills. A study of this topic can yield information on the best way to measure these constructs (for example using unidimensional or multidimensional scales) and also important design pointers for movement interaction applications.

We have called these constructs composite challenges because, as described above, they can be associated with the cognitive, physical or emotional parts of the person and therefore require several skills from the user. These challenges can be balanced when there is not a dominant element; and unbalanced when the challenge is mostly about one of its elements. For example: a game that requires the player to sit in front of the computer thinking and reacting to the actions (mental skills) using a minimum of physical movements, restricted to mouse and keyboard, is an unbalanced challenge. The hypothesis that motivates this study is that balanced challenges are more likely to promote flow episodes than unbalanced ones. The study reported in this paper evaluates this hypothesis empirically. In order to perform this evaluation, it compares a balanced with an unbalanced activity in terms of their potential to promote states of flow, operationalising flow as effortless attention.

\section{EVALUATING BALANCE IN COMPOSITE CHALLENGES}

The empirical study to evaluate the importance of balance in challenges took the form of an experiment in which participants were asked to play a movement interaction game and then evaluated their experience by answering a questionnaire.

\subsection{Experimental design}

The study comprised two parts, the first had a between subjects and the second a within subjects design. In the first part the independent variable was the type of composite challenge (a balanced or an unbalanced session), while the dependent variables were the players' change in state and their gaming experience (both of these self-reported using a questionnaire). In the second part the independent variable was the order of sessions (balanced first and unbalanced second or the other way round), while the dependent variable was the comparison of gaming experience (again self-reported with the use of a questionnaire). All significant results are reported with $p<0.05$.

The experimental session therefore combined a within with a between subjects design to ensure that the player's experience could be assessed from two perspectives: as an implicit comparison of the balanced and unbalanced tasks regarding their potential to promote flow states and as an explicit comparison in which the participants themselves could compare their playing experience over the two conditions.

\subsection{Procedure}

In line with this design the procedure of the study comprised two parts. In the first part participants were asked to fill in a questionnaire to record their state, then play the game (either the unbalanced or balanced version) and finally fill in the state questionnaire again plus a playing experience questionnaire. The motivation behind recording the players' state twice was to assess their change in state as a result of playing the game.

The second part took place immediately after the first one. In the second part participants were asked to play the game again but this time with the alternative version (balanced if the first one was unbalanced and vice versa). Finally they were asked to fill in a questionnaire that explicitly compared their playing experience over the two conditions. In both parts the gaming session lasted for about 10 minutes.

\subsection{Participants and materials}

There were 51 participants, 21 female $(41 \%)$ and 30 male $(59 \%)$. Participants were undergraduate students at two different universities (UNAM and UPSLP) and their ages ranged from 18 to 27 years. Most of them did not have any experience playing Kinect games and none had played the Postures game before.

Postures is a movement interaction game that was developed by the first author with the specific 


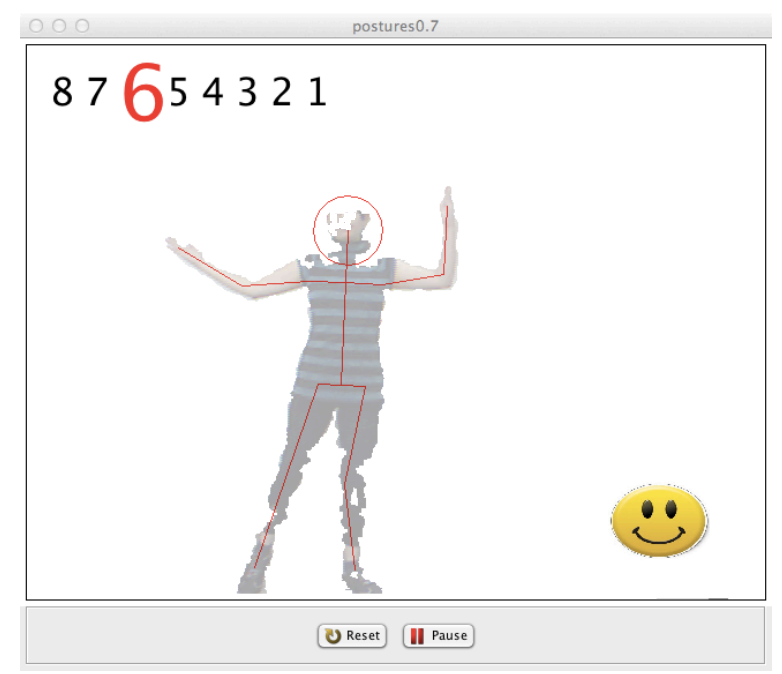

Figure 2: Screenshot of a gaming session with the Postures game.

purpose of performing the empirical study and that is played using Microsoft's Kinect device. In this game players have to imitate the postures of a stick-figure (see Figure 2). Postures are shown in sequences and are associated with the numbers appearing on the upper left corner of the game display. The number associated to the current posture appears on a larger, red font and is also called by the program. Players have a short span of time (a couple of seconds) to take the posture, after which the program evaluates it by showing either a happy, confused or sad face on the lower right corner depending on the player's performance. As with the posture number, there is a computer sound associated with each of these types of faces. Postures are shown immediately one after another for the duration of the playing session.

There were two versions of the game, an unbalanced and a balanced version. In the unbalanced version players had to imitate a set of postures (mainly a physical activity) while in the balanced version they had to also remember the set of postures (a mixture of physical and cognitive activities; after a certain period of training, the stick-figure would disappear but the program still expected the player to continue with the current posture in the sequence). The game evaluated how well the players performed a posture and adapted to their ability by giving them more time to take postures or more chances to learn them if they were unable to do so in the first place.

The questionnaires employed in the experiment contained questions commonly included in flow studies (Chen 2006; Csikszentmihalyi and Nakamura 2010; Finneran and Zhang 2005; Keller and Bless 2008). There were three questionnaires, the first one tried to ascertain the emotional state of participants, the second their playing experience and the third explicitly compared the playing experience over the two conditions. All of the questionnaires items had a Likert scale. The state questionnaire comprised items to assess the player's level of alertness, attention, awareness and happiness among other mood characteristics. The playing experience questionnaire comprised items to ascertain whether the player had experienced flow. Crucially, this questionnaire tried to assess whether the player's experience had been one of effortless or effortful attention by asking them how much did you concentrate? and how hard was it to concentrate? Additionally, the questionnaire also assessed the player's involvement, merging of action and awareness, level of excitement, selfconsciousness, enjoyment and the player's perception of the overall level of challenge of the game. Finally the comparison questionnaire was similar to the playing experience one but instead of asking for a single playing experience it asked the player to compare the two playing sessions (asking for example in which of the sessions did you concentrate the most? and in which of the sessions was it harder to concentrate?).

\section{RESULTS}

In line with the division of the questionnaire into three sections, the results of the study can be divided into those related to the players' state, those associated with their playing experience and those related to their comparison of the game versions. The analysis for the players' state compared their change in state (the difference between the before and after scores) for the two experimental conditions. All of the items registered a positive change (the after scores were consistently higher than the before scores), but there was no significant difference between the conditions for any of the items. This result suggests that although the players perceived an improvement in their state (they were more alert, more aware, more active, more energetic, more excited, more happy and more sociable after playing the game), the perceived magnitude of the improvement was similar for the two conditions.

Regarding playing experience, according to Csikszentmihalyi and Nakamura (2010), in a model assuming effortless attention the level of concentration should be inversely correlated with concentration effort and self-consciousness and directly correlated with involvement, merging of action and awareness, level of excitement and intrinsic motivation. We found this to be the case overall (when considering the data for both experimental conditions). The scores for the level of concentration (those associated with the question how much did you concentrate?) indeed correlated inversely with those of concentration effort 


\begin{tabular}{|c|c|c|}
\hline & & Concentration \\
\hline \multirow[t]{2}{*}{ Effort } & Correlation & $-.351^{*}$ \\
\hline & $\begin{array}{l}\text { Sig. } \\
\text { tailed) }\end{array}$ & .012 \\
\hline \multirow[t]{2}{*}{ Involvement } & Correlation & $.593^{*}$ \\
\hline & $\begin{array}{l}\text { Sig. } \\
\text { tailed) }\end{array}$ & .000 \\
\hline \multirow{2}{*}{$\begin{array}{l}\text { Merging of ac- } \\
\text { tion and aware- } \\
\text { ness }\end{array}$} & Correlation & $.435^{\star}$ \\
\hline & $\begin{array}{l}\text { Sig. } \\
\text { tailed) }\end{array}$ & .002 \\
\hline \multirow[t]{2}{*}{ Excitement } & Correlation & $.439^{*}$ \\
\hline & $\begin{array}{l}\text { Sig. } \\
\text { tailed })\end{array}$ & .002 \\
\hline \multirow{2}{*}{$\begin{array}{l}\text { Self- } \\
\text { consciousness }\end{array}$} & Correlation & $-.429^{*}$ \\
\hline & $\begin{array}{l}\text { Sig. } \\
\text { tailed })\end{array}$ & .002 \\
\hline \multirow{2}{*}{$\begin{array}{l}\text { Intrinsic motiva- } \\
\text { tion }\end{array}$} & Correlation & $.332^{*}$ \\
\hline & $\begin{array}{l}\text { Sig. } \\
\text { tailed) }\end{array}$ & .018 \\
\hline
\end{tabular}

Table 1: Pearson correlations for flow indicators. $N=51$, correlation is significant at the 0.05 level (*).

(those associated with the question how hard was it to concentrate) and with self-consciousness; and directly with involvement, merging of action and awareness, level of excitement and intrinsic motivation (see Table 1). When we investigated the correlations between level of concentration and concentration effort for each of the conditions (unbalanced and balanced sessions), we found the correlation to be significant only in the balanced case $(r(51)=-.492$, $\mathrm{p}<.05)$. This suggests that the balanced condition was responsible for the effect and therefore for the promotion of effortless attention in the game.

Additionally, the perceived level of challenge for both conditions was not significantly different from the commensurate level value (the middle value of the Likert scale), which means that participants overall did not think that the game was either too difficult or too easy.

Regarding the explicit comparison of playing experience, Figures 3 and 4 illustrate the comparisons for concentration level and concentration effort (associated with the questions in which of the sessions did you concentrate the most? and in which of the sessions was it harder to concentrate?, respectively). In both cases, the graphs represent the histograms of the 9 point likert-scale questionnaire items (which were normalised to the range -1 to 1 , with the middle point zero meaning no preference). In the case of concentration level (Figure 3 ), there were

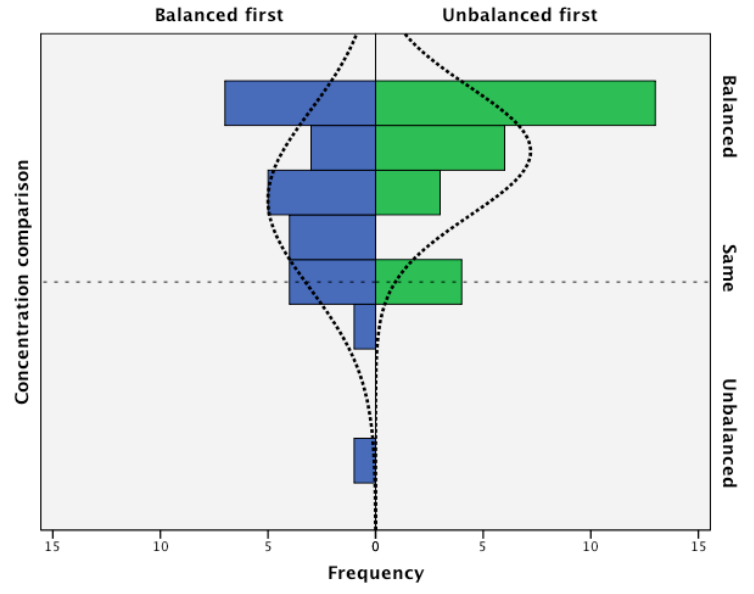

Figure 3: Comparison for level of concentration

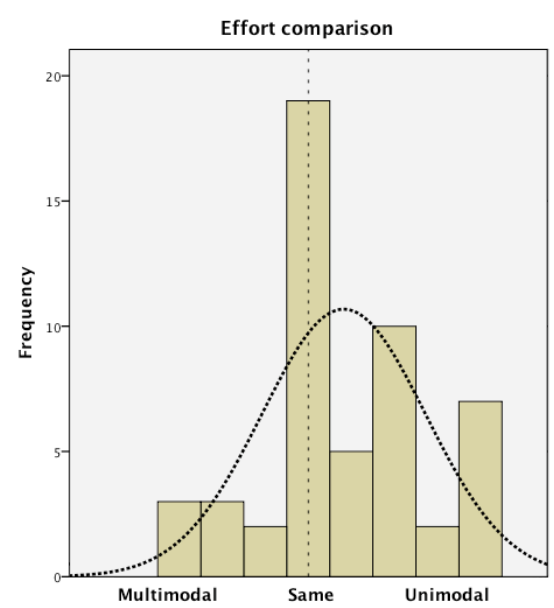

Figure 4: Comparison for concentration effort

significant differences between the conditions, this is, there were differences depending on which session was played first. The left hand side represents the graph for the balanced-first condition while the right hand side shows the unbalanced-first graph. In both cases, the upper part of the figure is for the balanced preference while the lower part is for the unbalanced. Although there were significant differences, both conditions had a preference for the balanced version, the only difference was that the unbalancedfirst condition had a stronger preference for that version. In other words, when asked in which game version did they concentrate the most, both those who started with the unbalanced version and those who began with the balanced version agreed that they concentrated the most with the balanced game $(\mathrm{M}=-0.598, \mathrm{SD}=0.45, \mathrm{t}(50), \mathrm{p}<.05)$. However those who started with the unbalanced version agreed more strongly $(F(1,49)=4.977, p<.05)$.

Regarding the concentration effort (Figure 4), there were no significant differences between the conditions, both of them had a preference for 
the unbalanced version $(M=0.205, S D=0.47, t(50)$, $\mathrm{p}<.05$ ). What this means is that both conditions agreed, to a similar extent, that it was harder to concentrate in the unbalanced version of the game.

\section{DISCUSSION}

This study evaluated the importance of balance in composite challenges by comparing a balanced with an balanced activity in terms of their potential to promote states of effortless attention or flow. The main result of the study suggests that the balanced activity promoted states of effortless attention and was considered as the one that promoted the highest level of concentration and the least level of concentration effort. This is in line with the conceptualisation of flow as effortless attention (Csikszentmihalyi and Nakamura 2010; Romero and Calvillo-Gamez 2011). These results were confirmed by two parts of the study, first by implicitly comparing the balanced and unbalanced tasks regarding their potential to promote flow states and second as an explicit comparison in which the participants themselves compared their playing experience over the two sessions of the game.

To the best of our knowledge, this study is the first to investigate balance in composite challenges within the context of flow. The findings of this study are important for at least three aspects: to support the embodied view of flow we have proposed (Romero and Calvillo-Gamez 2011), to refine the measurement of challenges and skills in flow studies and to guide the design of movement interaction games and applications.

The embodied view of flow we have proposed calls for a more complete view of interaction in studies of flow. Generally, studies of flow in computing have considered challenges and skills mainly as cognitive, perhaps because in traditional interaction the body has frequently not been taken into account (although human-computer interaction has always consisted of embodied action, mainly of small movements of the hands on the keyboard and mouse but embodied action nevertheless). The view of flow we propose emphasises the importance of the body; however, this importance does not lie on itself as a separate element but on its relationship with the intellectual part of the person. Therefore according to this view, an important characteristic of the challenges of the task is their balanced character, the fact that they have to take into account both the physical and intellectual parts of the person.

Another aspect of the embodied view of flow proposed that is supported by the results of this study is the conceptualisation and operationalisation of flow as effortless attention. As mentioned above, this is a characteristic that studies of flow in computing have ignored, however the results of this study suggests it is a central part of the concept. The fact that the level of attention was inversely correlated with the perceived effort and also correlated with the rest of the main characteristics of the flow state confirms that flow can be operationalised and conceptualised as effortless attention. Although the study by Csikszentmihalyi and Nakamura (2010) had already used a similar method to operationalise the concept, this is the first time this has been done in studies of flow in computing.

The findings of this study also suggest that the measurement of challenges should be refined. Traditionally, participants are asked whether they consider that the challenges of the task were commensurate with their skills. This is the only indication studies gather about the challenges-skills balance. Clearly, if challenges are balanced, it would make more sense to include questions about each one of the aspects challenges comprise as well as about its combined effect. For example, if the challenges of the task included psycho-motor coordination and mental arithmetic, questions about challenges should be of at least three types: those related with the psycho-motor coordination part, those associated with the mental arithmetic element and those that have to do with the combined effect of these two aspects.

Another way to take challenges into account in empirical studies is to include them as variables in the experimental design. The study by Keller and Bless (2008) is one of the few that have adopted this approach. In their study, they included three experimental conditions for three different levels of challenge: low, high and commensurate with the person's level of skill. Although this seems like a very sensible approach, if challenges are balanced, the experimental conditions would have to focus on specific aspects of challenges rather than on all of their elements at the same time. Using the same example as above, if the challenges of the task included psycho-motor coordination and mental arithmetic, the study might consider conditions such as low, high and commensurate psycho-motor coordination challenges; low, high and commensurate mental arithmetic challenges; and the combinations of those cases. Given that this would make the experimental design quite complex, it would be better to focus on one aspect at the time.

A practical application of the findings of the study has to do with the design of movement interaction systems concerned with promoting flow. According 
to the results of the study, these types of systems would be more efficient in promoting states of flow if they consider balanced rather than unbalanced activities. In line with our proposed view of flow, we hypothesise that integrating physical and psychic aspects in activities stretches people's abilities to its full capacity and in so doing an effortful and then hopefully an effortless period of attention is promoted. A further, important aspect to consider is about the integration of those aspects. In reality, almost any activity is balanced in the sense that it comprises several aspects and can be related with the physical, intellectual and emotional parts of the person. However, usually one aspect dominates and the others have only minimal participation. Two important questions regarding the combination of the elements of balanced challenges are concerned with their proportion of participation as well as with their appropriate integration. Regarding the first question, the assumption would be that a similar proportion of participation would be the best combination. This was what was intended in the design of the balanced challenge activity of the study reported here. However, this assumption has to be properly explored.

The second question has already been asked by Finneran and Zhang (2005). They have raised the issue of whether achieving a flow state depends on focusing all of the cognitive, physical and emotional parts of the person on the same activity. So it might not be enough to engage the different aspects of a person, what might be important is whether the different elements of a task are only tangentially related or closely integrated to the activity. For example, the balanced challenge of the activity in the study could have been associated with imitating the posture of the stick-figure plus keeping a count of random numbers appearing on the screen. Although this challenge has physical and intellectual aspects, they do not seem to be as well integrated to the activity as the one actually employed (imitating the posture plus remembering it). We hypothesise that the closer the integration of elements of challenges to the activity, the higher the likelihood of promoting flow states.

An additional factor to consider when designing flowinducing activities in movement interaction systems has to do with the aesthetic properties of movement. Bodily skill and movement in this type of interaction can have an aesthetic component and motor skills can also be a source of challenge and pride for users (Wensveen et al. 2004). Additionally, body movements can be used to modulate the user's experience through proprioceptive cues, for example requiring emotional and social expressive movements expressing the person's engagement in the task and with other agents involved in the task (Bianchi-berthouze ress). Exploiting movement in this way can be very important when trying to promote flow.

\section{CONCLUSION}

This paper has presented a study that evaluates the importance of balance in challenges associated with tasks designed to promote states of effortless attention or flow. The study suggests that balance is indeed an important aspect of this promotion. The main result of the study suggests that the balanced challenge activity promoted states of effortless attention and was considered as the one that induced the highest level of concentration and the least level of concentration effort. This result has important implications in supporting the embodied view of flow we have proposed elsewhere (Romero and Calvillo-Gamez 2011), as well as in refining the way challenges are measured in flow studies and to guide the design of movement interaction applications that aim to promote flow in users.

This study represents a first step in a project that is investigating balance in challenges within the context of an embodied view of flow. The next steps will consider a variety of challenge types, a closer look at the elements comprising challenges and into different ways of assessing the player's experience. The study considered a specific type of balanced challenge (kinesthetic memory). Different types of challenges might have greater or lesser potential to promote flow states. Also, balanced has been limited to physical and intellectual aspects in this study. A question worth pursuing is whether there are other aspects, for example affective or emotional, and how can they be included in the design of a digital application. A further issue relates to method and measurement. Traditionally, flow studies have employed selfreporting methods like questionnaires; however other methods, physiological correlates for example, have already been used in flow studies (Manzano et al. 2010). Employing physiological correlates methods seem quite appropriate in the context of investigating balance in challenges.

\section{REFERENCES}

Bianchi-berthouze, N. (In press). Understanding the role of body movement in player engagement. Human Computer Interaction.

Bruya, B. (2010). Introduction: Toward a theory of attention that includes effortless attention. In Bruya, B., editor, Effortless Attention: A New Perspective in the Cognitive Science of Attention and Action, pages 1-28. MIT Press. 
Chen, H. (2006). Flow on the net-detecting web users' positive affects and their flow states. Computers in Human Behavior, 22(2):221-233.

Csikszentmihalyi, M. (1990). Flow: The Psychology of Optimal Experience. Harper and Row, New York, NY.

Csikszentmihalyi, M. and Nakamura, J. (2010). Effortless attention in everyday life: A systematic phenomenology. In Bruya, B., editor, Effortless Attention: A New Perspective in the Cognitive Science of Attention and Action, pages 179-189. The MIT Press, Cambridge, MA.

Dourish, P. (2001). Where the Action Is: The Foundations of Embodied Interaction. The MIT Press, Cambridge, MA.

Ellis, G., Voelkl, J., and Morris, C. (1994). Measurement and analysis issues with explanation of variance in daily experiences using the flow model. Journal of Leisure Research, 26(4):337-356.

Finneran, C. M. and Zhang, P. (2005). Flow in computer-mediated environments: Promises and challenges. Communications of the Association for Information Systems, 15(4):82-101.

Hoffman, D. L. and Novak, T. P. (2009). Flow online: Lessons learned and future prospects. Journal of Interactive Marketing, 23(1):23-34.

Keller, J. and Bless, H. (2008). Flow and regulatory compatibility: An experimental test of the flow model of intrinsic motivation. Personality and Social Psychology Bulletin, 34:196-209.

Manzano, Ö., Theorell, T., Harmat, L., and Ullén, F. (2010). The psychophysiology of flow during piano playing. Emotion, 10(3):301-311.

Nakamura, J. and Csikszentmihalyi, M. (2002). The concept of flow. In Snyder, C. R. and Lopez, S. J., editors, Handbook of positive psychology, pages 89105. Oxford University Press, Oxford, UK.

Norman, D. A. and Shallice, T. (1986). Attention to action: willed and automatic control of behavior. In Davidson, R. J., Schwartz, G. E., and Shapiro, D., editors, Consciousness and Self-Regulation, pages 1-18. Plenum Press.

Novak, T. P., Hoffman, D. L., and Duhachek, A. (2003). The influence of goal-directed and experiential activities on online flow experiences. Journal of Consumer Psychology, 13(1-2):3-16.
Romero, P. and Calvillo-Gamez, E. H. (2011). Towards an embodied view of flow. In Ricci, F., Semeraro, G., d. G., M., Lops, P., Masthoff, J., Grasso, F., and Ham, J., editors, Proceedings of 2nd Workshop on User Models for Motivational Systems: The affective and the rational routes to persuasion (UMMS 2011), volume 740 of CEUR Workshop Proceedings, ISSN 1613-0073, pages 100-105.

Schmeichel, B. J. and Baumeister, R. F. (2010). Effortful attention control. In Bruya, B., editor, Effortless Attention: A New Perspective in the Cognitive Science of Attention and Action, pages 29-50. MIT Press, Cambridge, MA.

Schneider, W. and Shiffrin, R. M. (1977). Controlled and automatic human information processing: I. detection, search, and attention. Psychological Review, 84(1):1-66.

Shin, N. (2006). Online learner's flow experience: An empirical study. British Journal of Educational Technology, 37(5):705-720.

Skadberg, X. Y. and Kimmel, J. R. (2004). Visitors' flow experience while browsing a web site: its measurement, contributing factors and consequences. Computers in Human Behavior, 20(3):403-422.

Smallwood, J., McSpadden, M., and Schooler, J. W. (2007). The lights are on but no one's home: metaawareness and the decoupling of attention when the mind wanders. Psychonomic Bulleting \& Review, 14(3):527-533.

Smallwood, J. and Schooler, J. W. (2006). The restless mind. Psychological Bulletin, 132(6):946958.

Varela, F. J., Thompson, E., and Rosch, E. (1991). The embodied mind: Cognitive science and human experience. MIT Press, Cambridge, Massachusetts. Voiskounsky, A. E. (2008). Flow experience in cyberspace: Current studies and perspectives. In Barak, A., editor, Psychological Aspects of Cyberspace: Theory, Research, Applications, pages 70-101. Cambridge University Press, New York.

Wensveen, S. A. G., Djajadiningrat, J. P., and Overbeeke, C. J. (2004). Interaction frogger: a design framework to couple action and function through feedback and feedforward. In Proceedings of the 5th conference on Designing interactive systems: processes, practices, methods, and techniques, pages 177-184, Cambridge, MA, USA. ACM. 